\title{
NonDestructive High-Sensitivity Detections of Metallic Lithium Deposited on a Battery Anode Using Muonic X-rays
}

Izumi Umegaki $^{* a}$, Yuki Higuchi ${ }^{a}$, Yasuhito Kondo ${ }^{a}$, Kazuhiko Ninomiya $^{b}$, Soshi Takeshita $^{c, d}$, Motonobu Tampo $^{c, d}$, Hiroyuki Nakano $^{a}$, Hideaki Oka ${ }^{a}$, Jun Sugiyama ${ }^{a, c, d, e, f}$, Michael K. Kubo ${ }^{g}$, and Yasuhiro Miyake ${ }^{c, d}$

${ }^{a}$ Toyota Central Research \& Development Laboratories, Inc., Yokomichi, Nagakute, Aichi 480-1192, Japan

${ }^{b}$ Graduate School of Science, Osaka University, Machikaneyama, Toyonaka, Osaka 560-0043, Japan

${ }^{c}$ Muon Science Laboratory, High Energy Accelerator Research Organization (KEK), Tsukuba, Ibaraki 3050801, Japan

${ }^{d}$ Muon Science Section, Materials and Life Science Division, J-PARC Center, Tokai, Ibaraki 319-1195, Japan

${ }^{e}$ Advanced Science Research Center, Japan Atomic Energy Agency (JAEA), Tokai, Ibaraki 319-1195, Japan

${ }^{f}$ CROSS Neutron Science and Technology Center, Tokai, Ibaraki 319-1106, Japan

${ }^{g}$ College of Liberal Arts, International Christian University, Osawa, Mitaka, Tokyo 181-8585, Japan

*Email: umegaki@mosk.tytlabs.co.jp

\section{Table of Contents}

- P S2 - Text S-1 : Information of active anode material

- P S2 - Text S-2 : Sample preparation protocol

- P S3 - Figure S-1 : Charge and discharge curves in preparation of samples

- P S4 - Figure S-2 : Photographs, the SEM images, and ESR spectra

- P S5 - Table S-1: Conditions and assessment for samples

- P S6 - Figure S-3 : Compositions of the samples

- P S7 - Text S-3 : Comments for laminated samples

- P S7 - Text S-4 : Confirmation of the existence of Li metal deposition 


\section{Text S-1. Active anode material}

Natural graphite (OMAC2, Osaka Gas Chemical) was used for the active anode material. A slurry of $90 \mathrm{wt} . \%$ natural graphite and $10 \mathrm{wt} . \%$ polyvinylidene fluoride (PVdF) was coated onto a $\mathrm{Cu}$ foil $(10 \mu \mathrm{m}$ thick $)$. This coated sheet was dried and pressed to a thickness of $87 \mu \mathrm{m}$. The surface and bulk densities of the anode were $0.012 \mathrm{~g} / \mathrm{cm}^{2}$ and $1.38 \mathrm{~g} / \mathrm{cm}^{3}$, respectively. Graphite $\mid$ Li metal cells were fabricated in an Ar-filled glove box at Toyota CRDL. The electrolyte was $1 \mathrm{M} \mathrm{LiPF}_{6}$ dissolved in a mixed organic solvent, EC:DMC:EMC = 3:4:3. The separator was a porous polyethylene film, which was soaked in the electrolyte in the cells.

\section{Text S-2. Sample preparation in electrochemical measurements}

First, the cell was charged using a constant current (CC) from 0 to $5 \mathrm{mV}$ with a current density $(I)$ of $2.65 \mathrm{~mA} / \mathrm{cm}^{2}$, which was maintained for $2 \mathrm{~h}$. The cell was then discharged with $I=2.65 \mathrm{~mA} / \mathrm{cm}^{2}$ to $2.5 \mathrm{~V}$ and maintained at this voltage for $2 \mathrm{~h}$. Second, the cell was charged using a constant current constant voltage (CCCV) mode at $I=2.65 \mathrm{~mA} / \mathrm{cm}^{2}$ down to $5 \mathrm{mV}$ and maintained at $5 \mathrm{mV}$ for $2 \mathrm{~h}$ [Fig. S-1 (a)]. Then, the cell was covered with an $\mathrm{Al}$ foil and external short-circuited at $23^{\circ} \mathrm{C}$ for one week to obtain a fully charged graphite anode, $\mathrm{C}_{6} \mathrm{Li}$ (charged anode, sample $\mathrm{A}$ ). The color of sample A was yellow gold [Fig. S-2 (a)], which indicates the typical appearance of $\mathrm{C}_{6} \mathrm{Li}$.

Overcharged anodes were prepared by further charging sample A using a CC condition at $I=2.57 \mathrm{~mA} / \mathrm{cm}^{2}$, resulting in Li metal deposition onto the anode (samples B and C) [Fig. S-1 (b)]. The overcharged capacity of each sample was 16 and $32 \%$, respectively. The surface of sample $\mathrm{B}$ was covered with a thin white layer over the yellow basement [Fig. S-1 (b)]. The appearance of the surface of sample C was nearly identical to that of sample B. In addition to samples B and C, we fabricated a stacked sample (sample D) to change the depth of the deposited Li metal from the surface. In sample $\mathrm{D}, \mathrm{C}_{6} \mathrm{Li}$ with a thickness of $89 \mu \mathrm{m}$ lies on the top of sample $\mathrm{C}$ (Fig. S-3). 

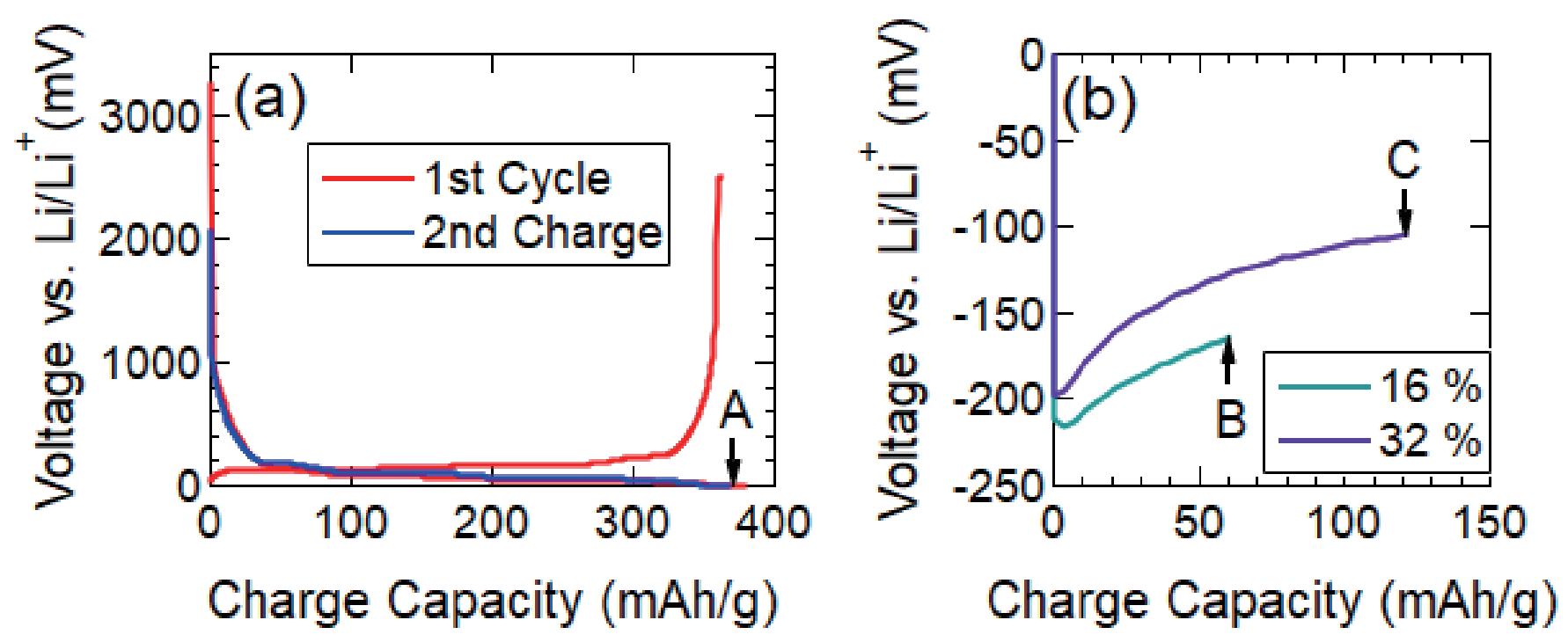

Figure S-1. The charge and discharge curves of the present samples for (a) the first charge and discharge cycle and second charge until $100 \%$ (sample A) and (b) the overcharge after the $100 \%$ charge. In (a), " $\mathrm{A}$ " indicates the $100 \%$ charge state. In (b), "B" and " $\mathrm{C}$ " represent the overcharge capacity for samples B and C, respectively. 

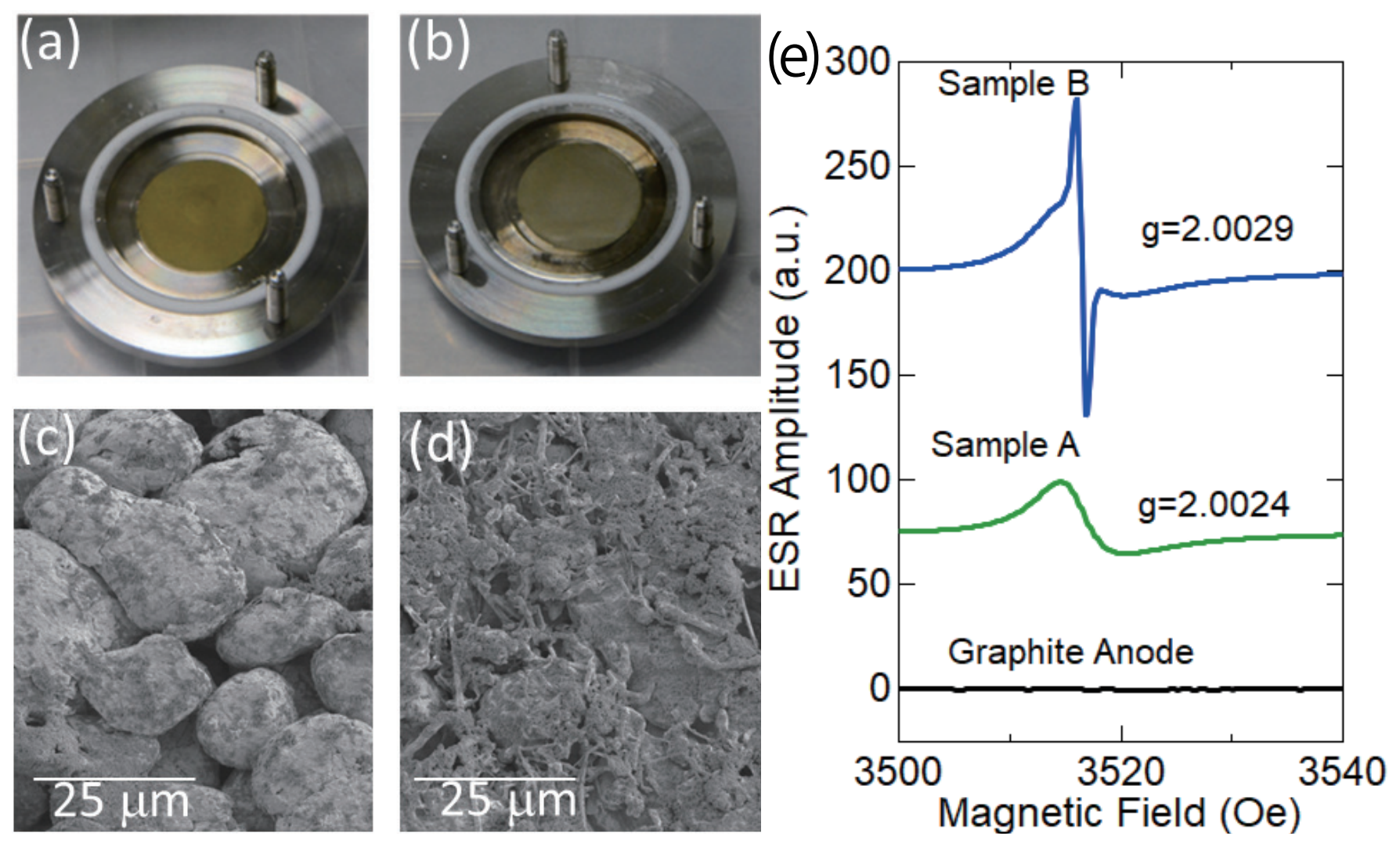

Figure S-2 : Photographs of the samples obtained in graphite | Li metal cells: (a) the fully charged graphite anode (sample A) and (b) the overcharged graphite anode (sample B). The SEM images of the surface of (c) sample A and (d) sample B, with an accelerating voltage of $1 \mathrm{kV}$. (e) The ESR spectra of sample A, sample B, and the graphite anode. The spectra of samples A and B are shifted upwards by 75 and 200 for clarity. The microwave output and frequency, module amplitude and frequency, and relaxation time were set as $0.625 \mathrm{~mW}$ and $9.6 \mathrm{kHz}, 1$ Oe and $100 \mathrm{kHz}$, and $30 \mathrm{~ms}$, respectively. The results of ten measurements were summed to obtain one spectrum. 


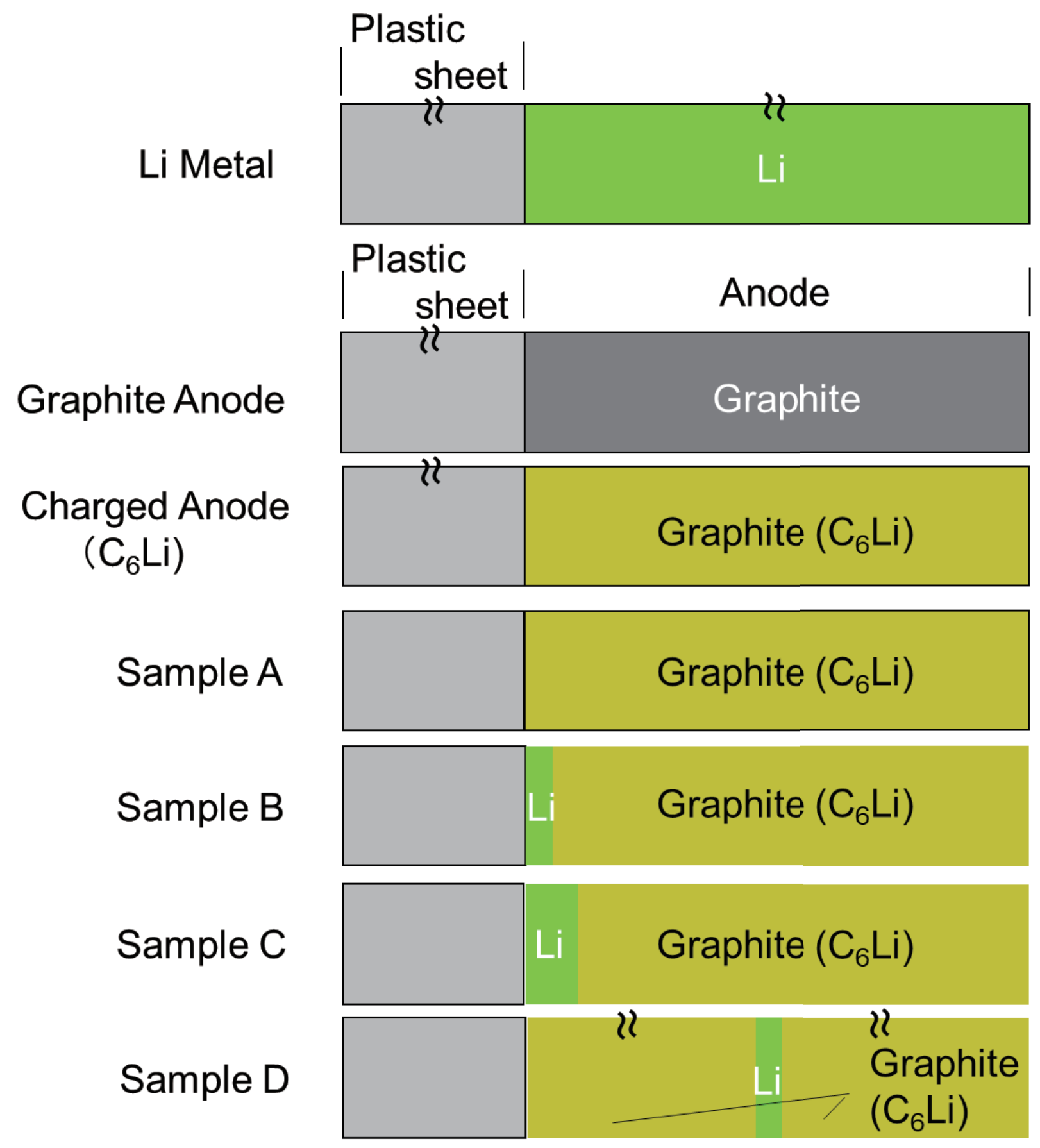

Figure S-3 : Schematic views of the compositions of the seven samples: Li metal, charged anode, graphite anode, and samples A, B, C, and D. 


\section{Text S-3. Laminated samples}

As the electrode samples are unstable in air, they were packed in an envelope made of an $\mathrm{Al}$ coated plastic sheet with a thickness of $44 \mu \mathrm{m}$, a plastic bag. In addition, a $\mathrm{Li}$ metal plate, with a thickness of $5 \mathrm{~mm}$ (Li metal), and an as-prepared graphite anode (graphite anode) were packed into a plastic bag for comparison.

\section{Text S-4. Confirmation of the existence of $\mathrm{Li}$ metal deposition}

The existence of Li metal deposition was confirmed using both SEM observations (S4300, Hitachi Hi-tech) and ESR analyses (ELEXYS E500, Bruker). Although the surface of sample A consists of graphite particles [Fig. S-2 (c)], it is very clear that needle-like compounds are deposited on the surface of sample B [Fig. S(d)]. Furthermore, the ESR spectrum of the graphite anode does not exhibit a resonance line while that of sample A exhibits an asymmetric Dysonian line shape, with $g=2.0024$. This line intensity is further enhanced in sample B [Fig. S-2 (e)] due to the metallic nature of the deposited $\mathrm{Li}{ }^{1,2}$, confirming the presence of Li metal.

The amount of Li metal was estimated using ICP-OES measurements (CIROS 120EOP, Rigaku). At first, the ICP-OES analyses showed that the amount of Li in sample A was $8.30 \mathrm{mg}$. In contrast, the amounts of $\mathrm{Li}$ in samples $\mathrm{B}$ and $\mathrm{C}$ were estimated as 9.55 and $11.41 \mathrm{mg}$, respectively, which led to 1.25 and $3.11 \mathrm{mg}$, respectively, for Li deposition by subtracting the amount of $\mathrm{Li}$ in sample $\mathrm{A}$ from those of samples B and $\mathrm{C}$. These results are consistent with estimations based on the charge and discharge capacity determined via electrochemical measurements.

\section{Reference}

(1) Pressley, R. J.; Berk, H. L. g Factor of Conduction Electrons in Metallic Lithium. Phys. Rev. 1965, 140, A1207-A1210.

(2) Dyson, F. J. Electron Spin Resonance Absorption in Metals. II. Theory of Electron Diffusion and the Skin Effect. Phys. Rev. 1955, 98 349-359. 